\title{
Bone lengthening with extra-articular arthrodesis of the hip using external fixation
}

\author{
Pedro Antonio Sánchez Mesa
}

Received: 30 December 2007/Accepted: 23 August 2008/Published online: 20 September 2008

(C) Springer-Verlag 2008

\begin{abstract}
This is a case series of 11 children and adolescents who underwent femoral lengthening and percutaneous hip arthrodesis using a method of extraarticular joint compression in combination with callotasis. Clinical review indicated a significant improvement in physical function and psychological well-being. The average lengthening was $5.3 \mathrm{~cm}$ or $16 \%$ of the initial length. Complications were more frequent in patients older than 14 years $(P=0.034)$ as was a higher maturation index $(P=0.021)$. No additional operations were required to achieve the surgical objective.
\end{abstract}

Keywords Arthrodesis · Extra-articular · Hip ·

Lengthening $\cdot$ Femur

\section{Introduction}

Femoral lengthening combined with hip arthrodesis is indicated for severe articular damage consequent to osteonecrosis; this may arise from sepsis, trauma, metabolic disorders or tumours. Lagrance in France in 1886 performed the first hip arthrodesis and since then many methods and techniques of surgical arthrodesis have been proposed. These methods can be classified in three categories: intra-articular, extra-articular and para-articular. These different techniques usually involve a wide surgical exposure, joint dislocation, articular debridement and in some cases peri-articular osteotomies, any or all of which

\section{P. A. S. Mesa ( $\square)$}

Clinic of Niño ISS "Jorge Bejarano" and Clinic 104 Saludcoop

"Jorge Piñeros", Calle 175 No. 17A-11(135), Bogotá, Colombia

e-mail: pasm@clubcadera.com

URL: www.clubcadera.com; www.clubrodilla.com may produce excessive bleeding [1-9, 12, 15, 19]. Stabilisation of the fusion in a position of $25^{\circ}$ flexion, $5^{\circ}$ abduction and $5^{\circ}$ external rotation is with internal or external fixation; this is sometimes supplemented with orthoses or plaster spicas. We present a case series of paediatric and young adult patients who have undergone an extra-articular arthrodesis of the hip and concurrent limb lengthening [10-14]. This technique utilises external fixation and is capable to avoiding plaster or hip-spica use, facilitates a more accurate position for arthrodesis, greater stability and consequently success in union, and an earlier return to functional activities. The technique described contrasts to traditional methods in which hip fusion is produced without formal hip joint exposure but through continuous articular compression with an external fixator and with simultaneous restoration of limb length equality by callotasis [16-19].

\section{Materials and methods}

This is a case series of 11 patients who were treated between January 2004 and November 2007 at the Paediatric Hip and Knee Service of the Orthopaedic and Traumatology Department. All presented with unilateral painful hips, secondary to articular destruction from various aetiologies, and were treated by extra-articular arthrodesis and concurrent limb lengthening. The variables recorded were age at surgery, diagnosis, the number of days for articular compression and osteotomy distraction, time for callus maturation, the total period of external fixator use and total treatment time.

Radiographs used for the purpose of this study were standardised. Bone length was measured before surgery and the amount of lengthening produced measured from 
the first radiograph of the maturation period. Any deformities over $10^{\circ}$ in the coronal plane were noted prior to fixator removal. Deformities in the sagittal plane were not recorded owing to the obstruction of view by the external fixator. Any deformities or fractures after fixator removal were also recorded.

The percentage of femoral lengthening was determined by dividing the amount of lengthening by the initial femoral length. The distraction rate was calculated from the length achieved divided by the number of days of distraction. A maturation index was obtained from dividing the duration of treatment (in days) by the lengthening achieved and expressed as either days per centimetre length or days percent elongation. These indexes were calculated because the femoral lengthening and the joint arthrodesis consolidated without internal fixation or bone grafting.

Indications for the procedure included tuberculous osteoarthritis, joint damage from septic arthritis, unilateral congenital hip dislocation presenting in the adolescent, sequelae of post-traumatic or degenerative osteonecrosis with absence of the ossific nucleus of the femoral head and of neck, congenital proximal focal deficiencies with femoral shortening greater than $13 \mathrm{~cm}$, and sequelae of slipped capital femoral epiphysis with severe coxa vara. Inclusion and exclusion criteria are listed in Table 1.

\section{Surgical technique}

For the bone elongation and arthrodesis procedure we use two monolateral external fixators-the Orthofix Modular System and Limb Reconstruction System (Orthofix SRL, Verona, Italy) (Fig. 1).

The patient is positioned on a radiolucent table and both limbs are prepped and draped. Antibiotic prophylaxis is used. An adductor tenotomy, performed through a groin crease incision, is carried out if hip abduction is noted to be less than $30^{\circ}$. In addition, tenotomies of the iliotibial band and hip flexors were carried out.

The fixator assembly for hip arthrodesis consists of a paediatric Dynamic Axial Fixator (DAF. Orthofix SRL,
Verona, Italy). First two tapered hydroxyapatite screws (length and diameter determined by age and quality of bone stock) are inserted into the supra-acetabular region using a T-clamp. These are angled $30^{\circ}$ in a cephalo-caudal direction and inserted percutaneously and with image intensifier control. This is followed by femoral diaphyseal screws which are placed perpendicular to the axis of the femoral shaft through the standard clamp of the DAF. The femoral diaphyseal screws are intentionally long (shaft portion) as a second Limb Reconstruction System rail fixator (LRS. Orthofix SRL, Verona, Italy) will be mounted over the same screws and positioned further lateral to the DAF. The fixator is adjusted through the central telescopic body and terminal ball joints in order to set the position of hip arthrodesis-usually in $20^{\circ}-30^{\circ}$ flexion, $0^{\circ}-15^{\circ}$ of external rotation and neutral abduction/adduction. The compression unit of the fixator is mounted across the central telescopic body and compression applied until there is sufficient evidence of contact of the articular surfaces.

An LRS rail fixator (usually $23 \mathrm{~cm}$ ) is then attached lateral ('outboard') to the initial paediatric DAF, sharing the same screws in the femoral diaphysis. The femoral fixation is then completed by adding two additional hydroxyapatite coated screws in the distal femur through the template of the LRS rail fixator. An osteotomy for lengthening is performed at the distal meta-diaphyseal junction using a percutaneous drill and osteotome technique (Figs. 2, 3). The wounds and pin sites are dressed and the limb bandaged.

The patient is discharged to out-patient (ambulatory) care when sufficiently recovered. In the first 2 weeks after surgery, compression of the hip is performed while adjusting for and maintaining the intended position for fusion. Femoral lengthening is commenced at day 15 postsurgery at an average rate of $1 \mathrm{~mm}$ a day (range 0.5$1.5 \mathrm{~mm}$ a day) by a quarter of turn of the distractor every $6 \mathrm{~h}$. A physiotherapy programme is started to maintain joint range and function in the limb. Prone lying is encouraged for $1-2 \mathrm{~h}$ per day in the first few days after surgery, and exercise and limb posture are monitored to avoid joint contractures. The exercise program is carried
Table 1 The following are criteria for the inclusion and exclusion of patients

\begin{tabular}{|c|c|}
\hline Inclusion criteria & Exclusion criteria \\
\hline $\begin{array}{l}\text { Patients with severe, unilateral, congenital or } \\
\text { acquired articular damage of the hip proven } \\
\text { clinically and radiologically }\end{array}$ & $\begin{array}{l}\text { Patients with bilateral hip involvement or } \\
\text { compromised neighbouring joints }\end{array}$ \\
\hline $\begin{array}{l}\text { Patients with severe functional limitation of one } \\
\text { limb with a visual analogue scale of pain }>8\end{array}$ & Patients with less than 3 months of observation \\
\hline Patients of ages $8-26$ years & Patients with ages outside the established range \\
\hline Patients with remaining normal joints & Chronic polyarthropathy \\
\hline $\begin{array}{l}\text { Patients with previously failed hip arthrodesis } \\
\text { (pseudoarthrosis) }\end{array}$ & Painful discitis or problems with the lumbar spine \\
\hline
\end{tabular}


Fig. 1 a Schematic drawing showing external fixator configuration. b The Orthofix (Verona SRL, Italy) modular dynamic axial fixator with T-clamp over the acetabulum. A LRS rail lengthener is coupled to the first fixator using extra-long screws inserted into the shaft of the femur a

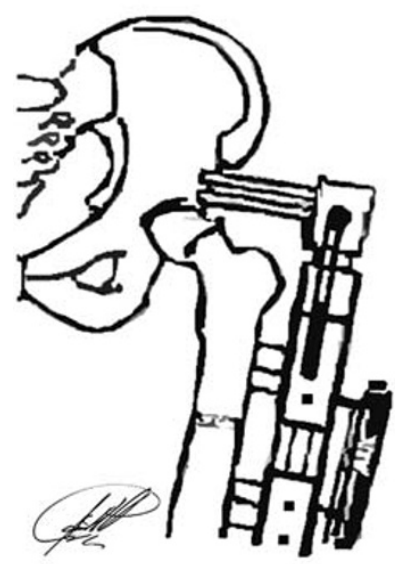

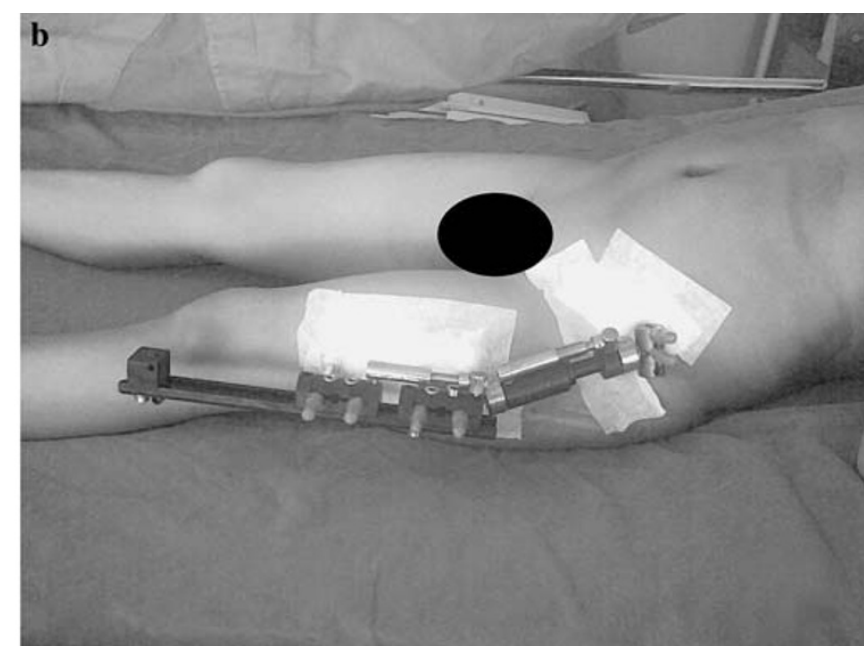

Fig. 2 a Image intensifier record of compression applied across hip during surgery. b Radiograph indicating regenerate length $(\mathrm{X})$ during process of limb reconstruction
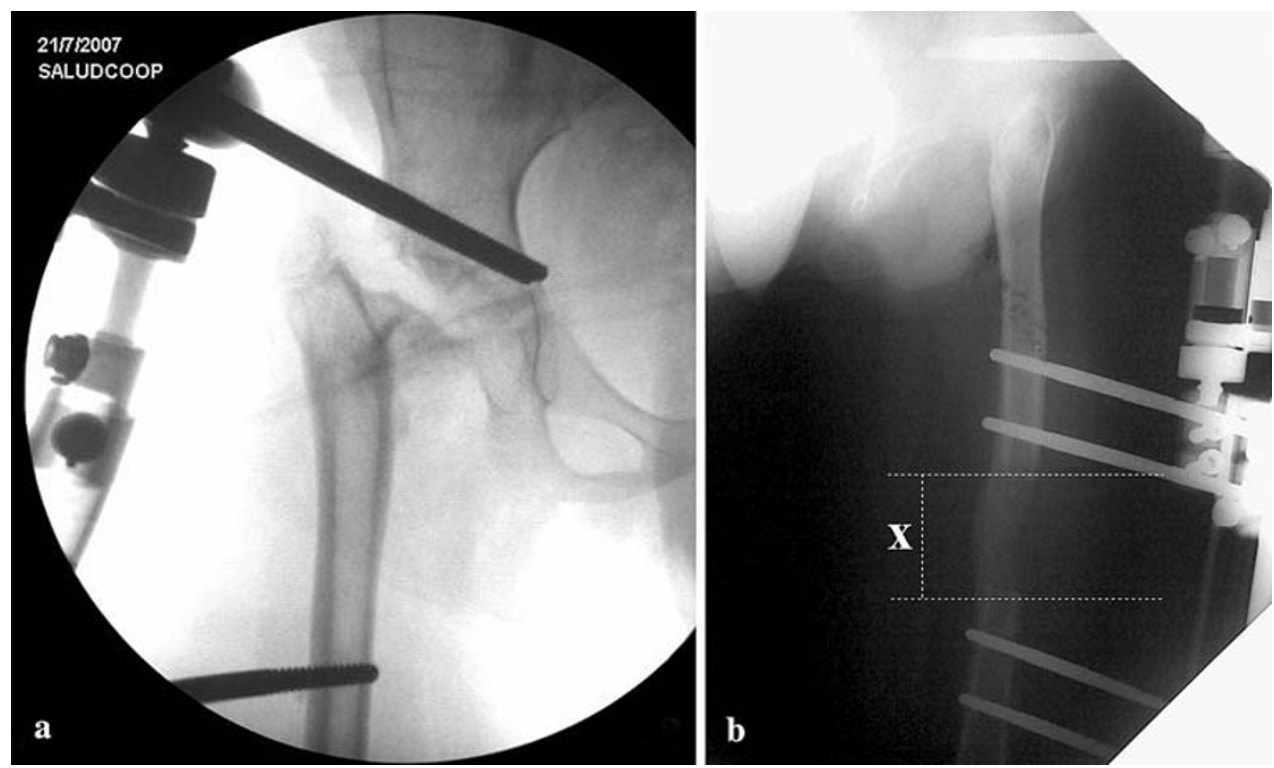

out three times a day, divided into static and dynamic types, with active quadriceps muscle contraction and mild bending of the knee over a straight edge coupled to passive manipulation and holding for $3 \mathrm{~s}$. This is repeated ten times in series of three until full flexion-extension of the knee is reached in the next 40 days. In addition, stretches of the hip flexors and dorsiflexion and plantar flexion of the ankle are practised (Fig. 4).

All patients attended weekly for clinical and radiological review during the period of lengthening and hip compression. Advice on maintaining knee extension and attendance to a regular physiotherapy programme was given. When the required lengthening was achieved, the external fixator was locked and a dynamiser (Dynaring. Orthofix SRL, Verona, Italy) inserted. Patients were also encouraged to increase their activity levels and weightbearing (Fig. 5). This period coincided with an emphasis on recovery of knee function and range of movement.
Some patients (older than 16 years) were provided additional stimulation through an electro-magnetic bracelet which was used for $1 \mathrm{~h}$ daily over the regenerate zone. On completion of treatment the external fixator and screws were extracted as an out-patient procedure in $80 \%$ of cases; this was carried out when three out of four cortices were visible within the regenerate column in AP and lateral radiographs. Plaster of Paris casts or orthotic splints were not used after fixator removal. Strengthening with physiotherapy was commenced 15 days after fixator removal.

\section{Statistical analysis}

We looked at the influence of the pre-operative and treatment variables on the number of interventions, maturation indices and complications. The patients were classified according to aetiology: group $\mathrm{A}$, congenital; group $\mathrm{B}$, 

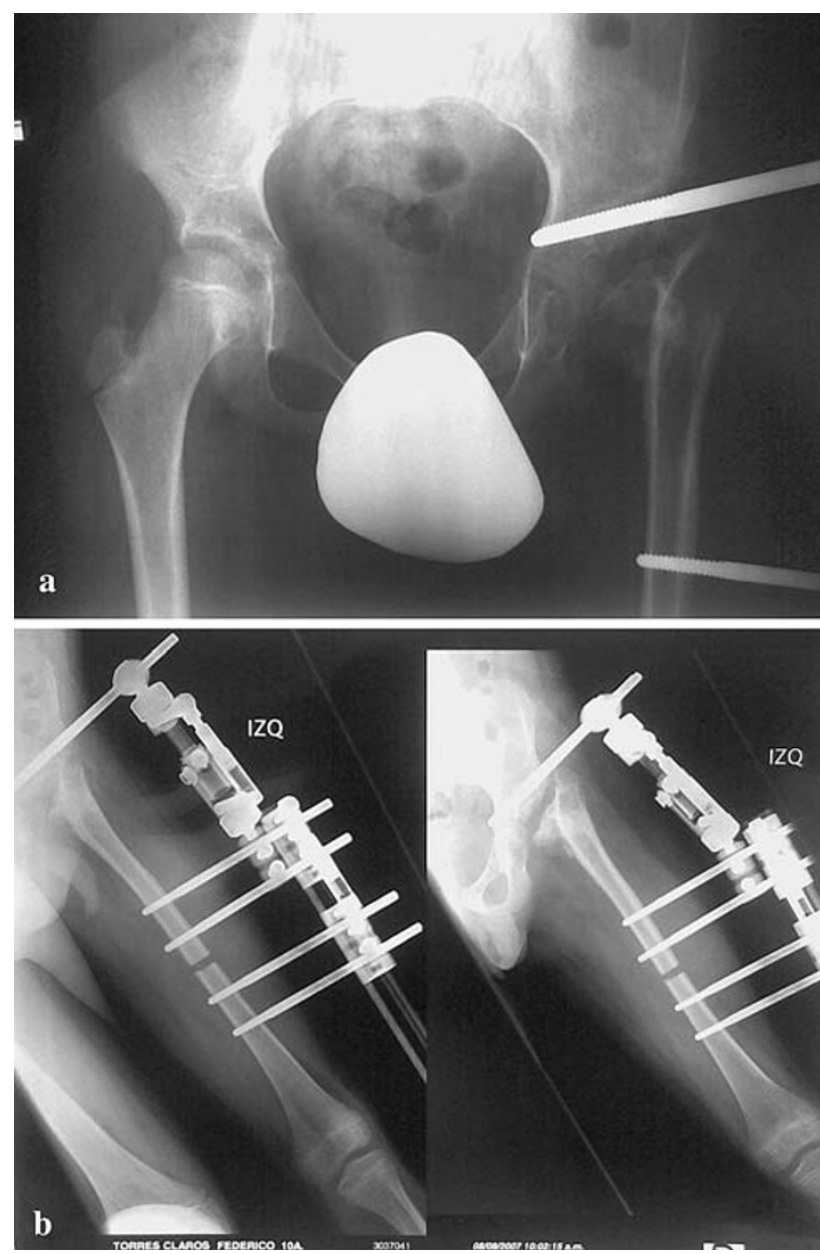

Fig. 3 a AP radiographs 1-week post-surgery showing position of arthrodesis and alignment of external fixator. b AP radiograph 2 weeks after lengthening

secondary aseptic osteonecrosis; and group C, secondary septic osteonecrosis. The pre-operative variables (independent) were age (grouped into those younger than 14 years and those older), diagnostic group, actual lengthening performed (grouped into those $<8,8-13$ and $>13 \mathrm{~cm})$, and percentage lengthening performed $(<25,25$ $60 \%$ and $>60 \%$ lengthening). The dependent variables included problems in regenerate formation (fractures, failure of formation), problems at the arthrodesis site (failure of fusion, need for further surgical intervention), and general complications (premature consolidation of the regenerate, deformity, osteolysis secondary to screw infection, neurovascular injury and additional operative interventions). The additional surgical interventions included osteoclasis for premature consolidation, manipulation of regenerate with or without fixator change for deformity, corrective osteotomies for residual angulation, arthrolysis, tenotomies for joint contractures, and bone graft with or without internal fixation for pseudoarthrosis of the regenerate column. These variables were regressed against the maturation index, time to fusion and percentage of length gained.

To assess the effect of the amount of lengthening on the incidence of complications and need for further surgery, divided the patients into subgroups based on the amount of lengthening performed. Subsequent regression analysis was carried out using the amount of lengthening as a covariate as this allowed the complication rate and need for additional surgery to be assessed against subgroups of lengthening. Univariate analysis of differences in observed and expected frequencies of complications was done using the Chi-squared test with the significance level set at $P<0.05$.

\section{Results}

There were complete data sets for 11 patients who underwent treatment of severe painful hip damage by arthrodesis and lengthening using external fixation. There was a minimum follow up of 3 months post-removal of external
Fig. 4 Stretching exercises program-basic postures and sleeping positions
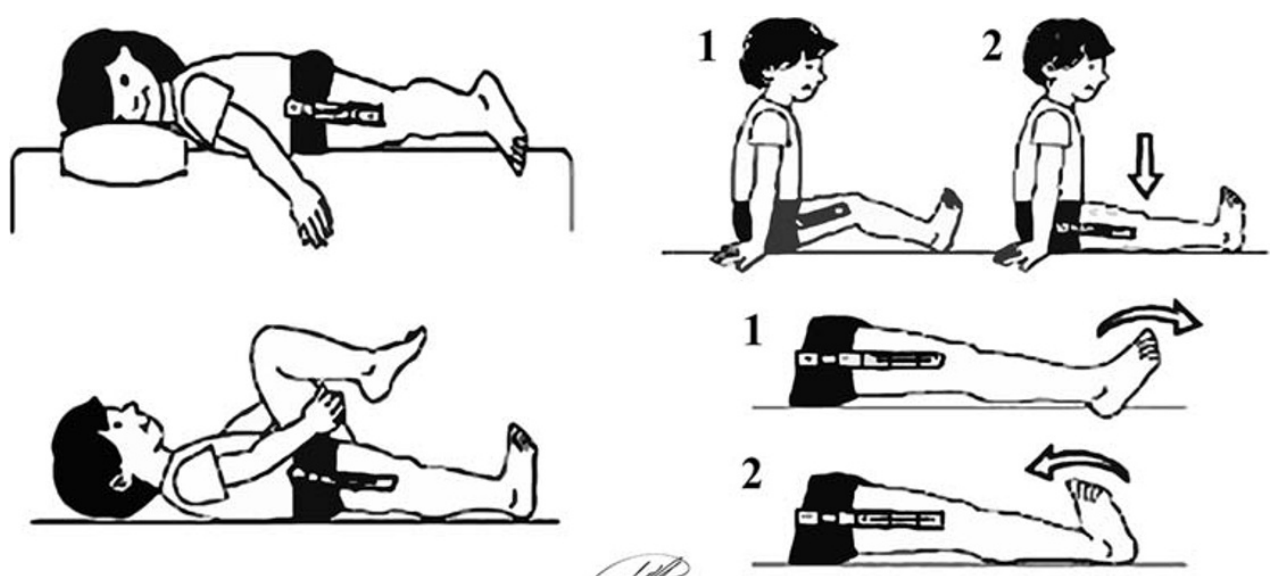
Fig. 5 a Arthrodesis of the hip now complete. b Final lengthening and percentage length gained. c A 11-year old child with limb length symmetry and post-hip arthrodesis
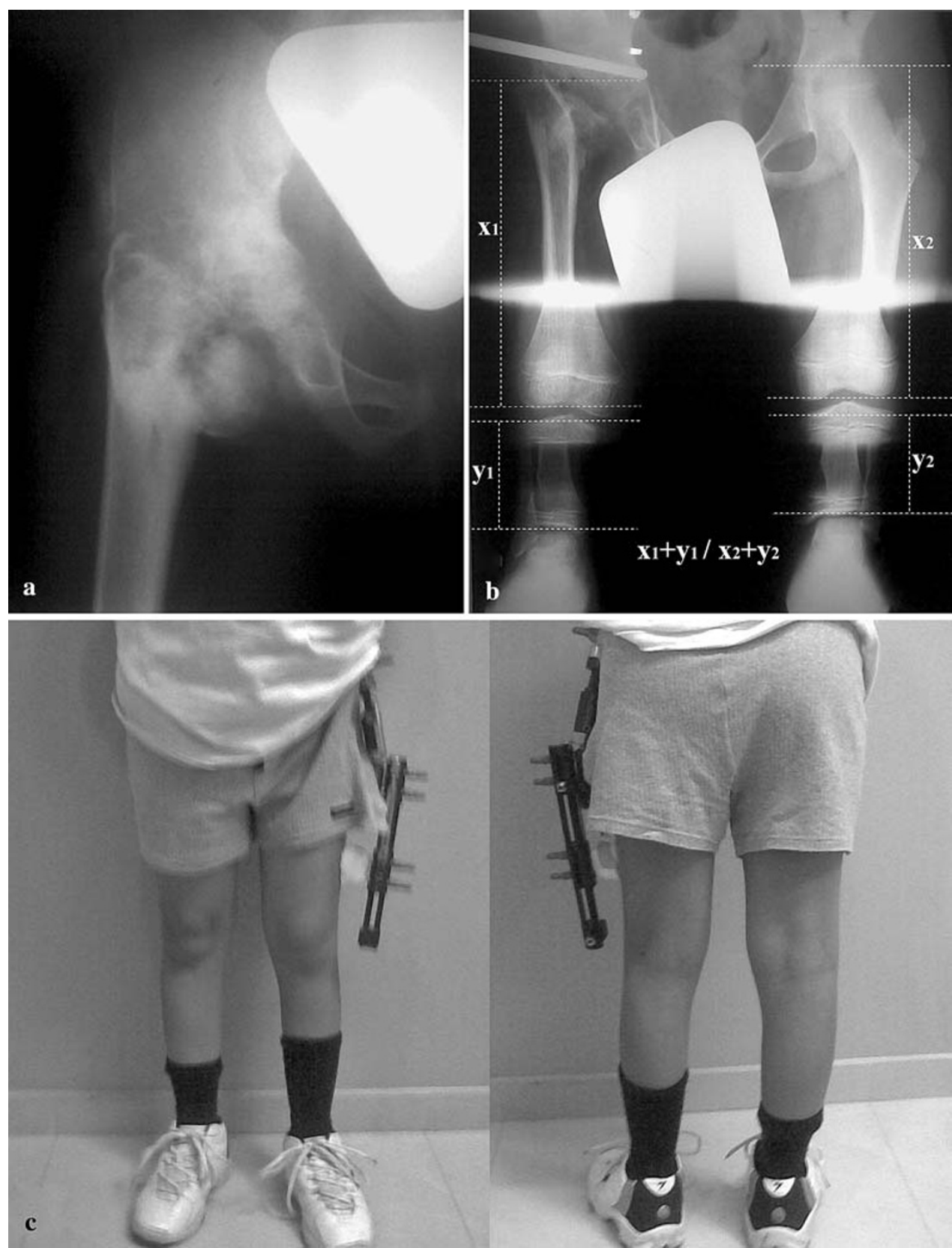

fixator. The mean age at surgery was 12.5 years (826 years); six were males and five females. Four cases (36.4\%) was due to post-traumatic changes of osteonecrosis, $3(27.3 \%)$ subsequent to sepsis (1 of which was tuberculous arthritis), three (27.3\%) from failed previous arthrodesis and one $(9.1 \%)$ degenerative ankylosis subsequent to treatment for congenital hip dislocation. Most patients had undergone an average of two operative procedures prior to the described technique; three patients had hip salvage using the procedure of trochanteric arthroplasty.

Arthrodesis in the described position was accomplished in all patients. This was confirmed clinically and radiologically. All patients reported an improvement in pain.
The mean period for fusion was 4.18 months (range 3-7) and this coincided with the period at which distraction for lengthening was completed (mean 98 days, range 82190 days). The average lengthening was $5.3 \mathrm{~cm}$ (range $3.5-17 \mathrm{~cm}$ ) which gave an average $48 \%$ increase in length of the femur (range 8.4-85.7\%). The total fixator time was 213 days (range 98-383 days) and treatment time 180 days (range 103-390 days). The average follow-up was 1.2 years (range 7 months to 3 years and 7 months). We noted a decrease in the maturation index with the longer femoral lengthenings. Patients who had undergone shorter femoral lengthenings had higher maturation indices $(P<0.001)$. 
Analysis of the maturation index did not show a statistically significant difference as a function of the diagnostic groups. Patients older than 14 years had higher maturation indices than younger ones $(P=0.002$ for the days $/ \mathrm{cm}$ index; $P=0.019$ for the days per $\%$ index $)$.

\section{Complications}

There were no neurovascular complications. There were no fractures or deformities associated with the regenerate column. Premature consolidation was noted in $29.7 \%$ but responded to increase in distraction rate and did not require osteoclasis or repeat osteotomy. No additional procedures were needed in the 11 cases (with the exception of fixator removal).

There were infected pin sites in two cases producing osteitis (18.1\%); the isolated bacteria were Staphylococcal aureus in one and polymicrobial in the other. Both responded to oral antibiotic therapy without need for screw exchange.

The frequency of complications and need for further surgery were analysed with respect to the magnitude of lengthening. There were no problems with the quality of the regenerate in those who underwent lengthening of less than $8 \mathrm{~cm}$ and in the $8-11 \mathrm{~cm}$ group (45 and 50\% of cases, respectively). This was statistically significant $(P=0.007)$ when compared to larger femoral lengthenings of 11$13 \mathrm{~cm}$ and those over $13 \mathrm{~cm}$ (no problems in 16 and $17 \%$, respectively). Although not statistically different, a similar trend was observed when patients were divided into those who underwent less than $45 \%$ lengthening to those over $45 \%$. There was also a major incidence of knee joint problems in those lengthened more than $13 \mathrm{~cm}$ or greater than $60 \%$ of the initial femoral length. There was a $63 \%$ incidence of joint problems in the group lengthened more than $13 \mathrm{~cm}$ compared to 19 and $40 \%$ in the subgroups with smaller lengthenings $(p=0.011)$. A similar finding was noted in comparing the patients who had greater than $60 \%$ lengthenings to those who had a smaller magnitude of procedure.

Patients with endocrine problems had a higher incidence of regenerate problems than those with a skeletal or growth dysplasia aetiology $(P=0.003)$. Age was also noted to have an influence with patients older than 14 years having a higher total incidence of complications $(P=0.047)$ than younger patients, especially in relation to knee joint problems and regenerate formation.

\section{Discussion}

This case series of extra-articular arthrodesis of the hip combined with simultaneous femoral lengthening reports outcome measures such as length produced through distraction osteogenesis, time to hip fusion, and the maturation index. The use of external fixation in conjunction with the technique of extra-articular arthrodesis obviates the need for hip joint exposure and represents a minimally invasive and biological procedure that also facilitates early ambulation and rehabilitation.

The maturation indices were related to the magnitude of lengthening, whether expressed in centimetres or as a percentage of the original femoral length [20-28]. When the length increased, the maturation index decreased. Although bone formation was independent of the magnitude of lengthening, there were greater complications in patients older than 14 years and in the longer lengthenings. However, this technique should be considered when the age of the patient, degree of articular damage, aetiology of the condition are such that arthroplasty and corrective osteotomies are not suitable options. The technique is relatively simple and hip fusion was achieved in a relatively short period in the desired position. This was possible through the facility to adjust the position of fusion after application of the external fixator. Additionally bio-compression through external fixation produced fusion without the need of bone grafts or rigid internal fixation devices. Complications such as pin site osteitis did not influence the final outcome with many patients reporting remission of pain from the hip.

\section{Summary}

We present a series of 11 patients with destructive hip conditions producing pain that were salvaged using an extra-articular arthrodesis technique by external fixation. Patients also underwent simultaneous femoral lengthening when significant shortening was a presenting feature. The technique is notable for achieving arthrodesis without formal exposure of the hip joint and providing the facility for substantial correction of leg length inequality. Maturation indices reduced with larger lengthenings but it was noted that knee joint problems and poorer regenerate quality were linked to these larger lengthenings in patients older than 14 years.

\section{References}

1. Calvache G, Satizabal C, Calderón O (2000) Artrodesis extraarticular de la cadera mediante fijacion externa para la coxartrosis dolorosa. Rev Colomb Ortop Traumatol 14(1):8-12

2. Sánchez M, Pedro A (2004) Manual Práctico para Residentes de Ortopedia Carbel. Bogotá, DC, Colombia

3. Guidera HJ, Hess WF, Highhouse KP, Ogden JA (1991) Extremity lengthening: results and complications with the Orthofix system. J Pediatr Orthop 11:90-94 
4. Britain HA (1941) Ischiofemoral arthrodesis. Br J Surg 29:93104

5. Ilizarov GA, Trohova VG (1973) Operative elongation of the femur. Orthop Traumatol Protez 34:51-55

6. Callaghan JJ, Penderson DR (1985) Hip arthodesis, a long term follow-up. J Bone Joint Surg 67A:1328-1355

7. Cañadell J, Aquerreta D, Forriol F (1993) Prospective study of bone lengthening. J Pediatr Orthop 2:1-7

8. Fulkerson JP (1997) Arthodesis for disabling hip pain in children and adolescents. Clin Orthop 128:296-302

9. Paley D (1988) Current techniques of limb lengthening. J Pediatr Orthop 8:73-92

10. Aldegheri R, Renzi-Brivio L, Agostini S (1989) The callotasis method of limb lengthening. Clin Orthop 241:137-145

11. Fernández Dell'Oca HA, Perren SM, Buchanan S (1992) External fixation with simple pin fixation. Injury 23:S11-S12

12. Rene L (1978) Hip arthrodesis and associated problems. Springer, Berlin

13. Lipscomb PR, Mc Castin FE Jr (1961) Arthrodesis of the hip. A review of 371 cases. J Bone Joint Surg 43A:923-938 934

14. Grill F, Dungl P, Steinwender G, Hosny G (1993) Congenital short femur. J Pediatr Orthop 2:335-341

15. Suziki S, Kasahara Y, Seto Y (1994) Dislocation and subluxation during femoral lengthening. J Pediatr Orthop 14:343-346

16. Sponseller PD, Mc Benth AA, Perpich M (1984) Hip arthrodesis in young patients. J Bone Joint Surg 66A:853-858

17. Eyres KS, Bell MJ, Kanis JA (1993) New bone formation during leg lengthening evaluated by dual energy X-ray absorptiometry. J Bone Joint Surg (B) 75:96-106
18. Stewart MJ, Coker TP (1950) Arthrodesis of the hip, a review of 109 patients. Clin Orthop 62:136-150

19. Stinchfield FE, Cavallaro WU (1950) Arthrodesis of the hip joint. A follow-up study. J Bone Joint Surg 32A:48-58

20. Tavares J, Frankovitch KF (1998) Hip arthrodesis using the AO modular external fixation. J Pediatr Orthop 18:651-656

21. Pouliquen JC, Ceolin JL, Lanlais J, Pauthier F (1993) Upper metaphyseal lengthening of the tibia by callotasis: forty-seven cases in children and adolescents. J Pediatr Orthop (B) 2:49-56

22. Thompson FR (1956) Combined hip fusion and subtrochanteric osteotomy allowing early ambulation. J Bone Joint Surg 38A:13-22

23. De Bastiani G, Aldegheri R, Brivio LR, Trivella G (1987) Limb lengthening by callus distraction (callotasis). J Pediatr Orthop $7: 129-134$

24. Eldrige JC, Bell DF (1991) Problems with substantial limb lengthening. Orthop Clin North Am 22(4):625-631

25. Karger C, Guille J, Bowen J (1993) Lengthening of congenital lower limb deficiencies. Clin Orthop 291:236-245

26. Aquerreta D, Forriol F, Cañadell J (1994) Complications of bone lengthening. Int Orthop (SICOT) 18:299-303

27. Glorion C, Pouliquen JC, Langlais J, Coelin JL, Kasis B (1996) Femoral lengthening using the callotasis method; study of the complications in a series of 70 cases in children and adolescents. J Pediatr Orthop 161:167

28. Fischgrund J, Paley D, Suter C (1994) Variables affecting time to bone healing during limb lengthening. Clin Orthop 301:31-37 